\title{
Retrieval of soil surface roughness from active and passive microwave observations
}

\author{
$\underline{\text { Y. Gao }}^{\text {a }}$, J. P. Walker ${ }^{\text {a }}$, R. Panciera ${ }^{\text {b }}$, A. Monerris ${ }^{\text {a }}$ and D. Ryu ${ }^{\text {c }}$ \\ ${ }^{a}$ Department of Civil Engineering, Monash University, Australia \\ ${ }^{b}$ Cooperative Research Centre for Spatial Information, University of Melbourne, Australia \\ ${ }^{c}$ Department of Infrastructure Engineering, University of Melbourne, Australia \\ Email: ying.gao@monash.edu
}

\begin{abstract}
Spatial and temporal variation in soil moisture plays a significant role in establishing efficient irrigation scheduling, climate change prediction, and sustainable land and water management. Passive microwave remote sensing at L-band is widely recognised as the preferred technique to measure surface soil moisture globally, with spatial resolution ranging from 40-100km. However, passive microwave soil moisture retrieval is highly dependent on ancillary data such as surface roughness, which is difficult to characterise by ground measurement. The National Aeronautics and Space Administration (NASA) is developing the Soil Moisture Active Passive (SMAP) mission, scheduled for launch at the end of October 2014. SMAP will deploy both L-band active (radar) and passive (radiometer) microwave instruments to enhance the soil moisture retrieval capabilities. Consequently, deriving roughness information from the active microwave sensor onboard SMAP provides an opportunity to solve the aforementioned problem. However, it is unclear if roughness parameters derived from active microwave data can be used directly in passive microwave retrievals.
\end{abstract}

This paper presents a series of roughness-related analysis using data from the Soil Moisture Active Passive Experiment (SMAPEx), conducted in south-eastern Australia in 2010-2011. The SMAPEx airborne instrumentation comprised an L-band radar (PLIS) and radiometer (PLMR), replicating the active/passive configuration of the SMAP satellite. Intensive ground soil moisture sampling was undertaken concurrently with each flight, and surface roughness was sampled within each major land cover type. In this study, three $1 \mathrm{~km}^{2}$ pixels with relatively homogeneous bare surface were selected, representing three types of roughness patterns: sinusoidal, flat bench and non-periodic structure. Both soil moisture and roughness parameters of these three pixels were retrieved from PLIS backscatter coefficient and PLMR brightness temperature respectively, and then compared with each other.

Results show that soil moisture values retrieved from passive measurements have a much higher accuracy $\left(\mathrm{RMSE}=0.05 \mathrm{~m}^{3} / \mathrm{m}^{3}\right.$ ) than those retrieved from active measurements $\left(\mathrm{RMSE}=0.11 \mathrm{~m}^{3} / \mathrm{m}^{3}\right)$. Active microwave significantly underestimated the soil moisture (Bias $=0.76 \mathrm{~m}^{3} / \mathrm{m}^{3}$ ), especially in the top soil moisture range $\left(0.15-0.35 \mathrm{~m}^{3} / \mathrm{m}^{3}\right)$. In addition, the studied pixel with non-periodic structure showed the best roughness retrieval accuracy $(\mathrm{RMSE}=0.35 \mathrm{~cm})$ from active measurements compared with ground measurments, while the algorithm tends to overestimate the roughness at the two pixels with periodic structure (RMSE $=0.52 \mathrm{~cm}$ and $0.63 \mathrm{~cm}$ respectively). Importantly, it was found that the roughness parameter $H_{R}$ retrieved from passive microwave was higher than $H_{R}$ retrieved from active microwave for all pixel types (calculated using two different formulations from retrieved surface root-mean-square height, Bias $=0.21$ and 0.36 respectively), leading to the conclusion that passive microwave may be more sensitive to larger-scale of roughness than active microwave. However, these results still need to be confirmed with more data points.

Keywords: soil moisture; surface roughness; Soil Moisture Active Passive Experiment (SMAPEx); active microwave; passive microwave; RMS height 


\section{INTRODUCTION}

Soil moisture plays a fundamental role in land surface hydrology, as it controls the exchange of water and heat energy between the land surface and the atmosphere through evaporation and plant transpiration. Passive microwave remote sensing provides a unique capability for direct observation of soil moisture, especially for frequent, global sampling of soil moisture over a large fraction of land surface (Njoku and Entekhabi, 1996). Research activities carried out worldwide in the past decade have proved that low-frequency $(1-3 \mathrm{GHz})$ microwave is considered optimum for soil moisture monitoring due to the reduced atmospheric attenuation and greater vegetation penetration. The Soil Moisture and Ocean Salinity (SMOS) mission from the European Space Agency (EAS), launched in November 2009 carrying an L-band interferometric radiometer, was the first soil-moisture dedicated passive microwave mission. The multi-incidence angle and dualpolarization capabilities of the SMOS radiometer allow novel approaches for retrieval of the top $0-5 \mathrm{~cm}$ soil moisture, with a revisit time of 2-3 days and a spatial resolution of $40-\mathrm{km}$ resolution globally (Kerr et al., 2010).

In order to retrieve soil moisture from passive microwave, information on the land surface characteristics is required. At L-band, vegetation water content and soil surface roughness significantly impact the surface emission for a given soil moisture condition (Panciera et al., 2009). However, ancillary information such as surface roughness is difficult to characterise, especially at large scale over the Earth's surface. Up to now, most implementations of the soil moisture retrieval algorithms have applied a default roughness parameter, or calibrated the roughness parameter according to the land cover (eg. Wigneron et al., 2007; Saleh et al., 2009). The possibility to derive roughness parameter from active microwave (radar) measurements and to apply it to the retrieval of soil moisture from passive microwave (radiometer) measurements has not yet been thoroughly studied. As with passive microwave remote sensing, the variations in radar backscattering are also influenced by soil moisture, surface roughness, vegetation cover and so on. However, compared with passive microwave, active microwave sensors are more sensitive to surface roughness, even more sensitive than to soil moisture in most cases (Schmugge, 1985). Thus, active microwave measurements provide an opportunity to characterise surface roughness, and thus improve the passive soil moisture retrieval accuracy.

The upcoming National Aeronautics and Space Administration (NASA) soil moisture dedicated mission SMAP (Soil Moisture Active Passive), scheduled for launch in 2014, will carry both an L-band radar and radiometer (Entekhabi et al., 2010). SMAP will use the synergy between active and passive measurements to undertake downscaling of the brightness temperature. This also provides an opportunity to enhance soil moisture retrieval capabilities in relation to roughness. However, it remains unclear whether the roughness parameters derived from active microwave data can be used directly in passive microwave retrievals. It suffers from at least two problems: 1 ) active and passive measurements have different spatial resolution (3km and $40 \mathrm{~km}$ respectively for SMAP); and 2) the roughness value retrieved from active measurements has a different physical meaning to the roughness parameter input required by the passive microwave soil moisture retrieval model. While in active microwave roughness is usually characterized by surface Root Mean Square (RMS) height, roughness in passive microwave is described using a parameter $H_{R}$, which is an 'effective' parameter and not physically measurable (Choudhury et al., 1979). Consequently, this study performs a comparison between surface roughness parameters retrieved from active and passive measurement over bare soils using data from the third Soil Moisture Active Passive Experiment (SMAPEx-3) conducted in southeastern Australia in 2011.

\section{DESCRIPTION OF DATA SET}

The SMAPEx-3 field experiment was conducted in the Yanco study area within the Murrumbidgee catchment in south-eastern Australia from 5 to 23 September, 2011 (Panciera et al., 2013). It aimed to provide both airborne active and passive microwave data supported by ground sampling of soil moisture, vegetation water content and soil roughness. Airborne data were collected over an area equivalent to a single SMAP radiometer pixel $(36 \mathrm{~km} \times 38 \mathrm{~km})$ for a total of 9 dates over a 3-week period. Intensive spatial monitoring was conducted at six focus areas equivalent to a SMAP radar pixel $(3 \mathrm{~km} \times 3 \mathrm{~km})$. Being in the Austral spring, this campaign commenced with moist soils and low vegetation biomass, leading to dry soils and high vegetation biomass towards the end of the experiment. In this study, three $1 \mathrm{~km}$ pixels with relatively homogeneous bare surface were selected from two of the six focus areas, YA4 and YA7 [see Figure 1(a)]. These pixels also represent three types of roughness: sinusoidal, flat bench and non-periodic structure [see Figure 1(b)]. The soil was classified as silty clay loam according to the soil texture analysis. 
(a)

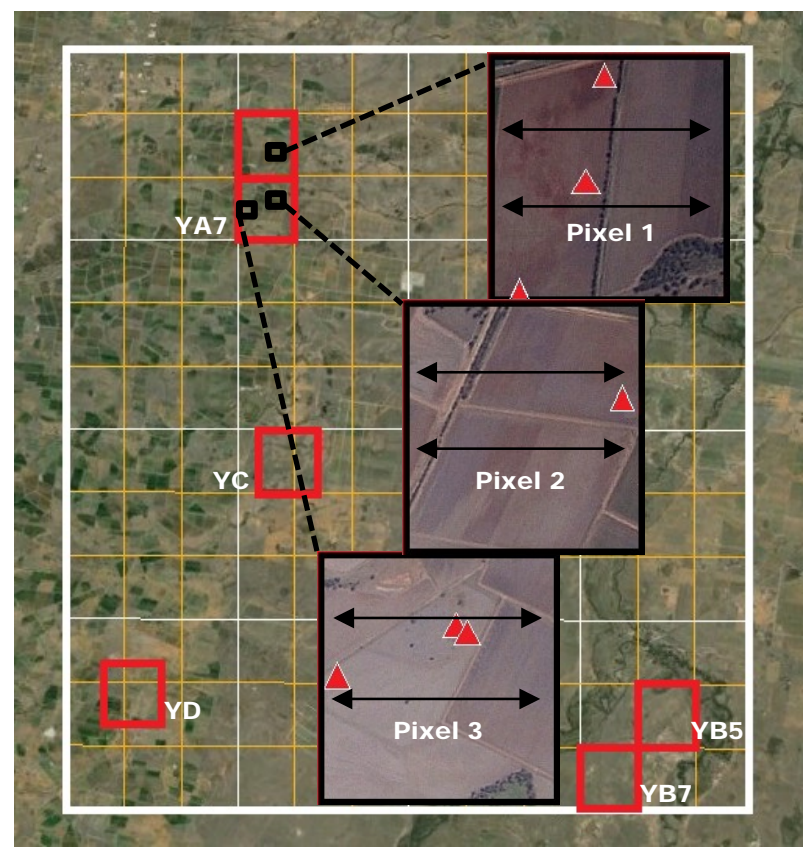

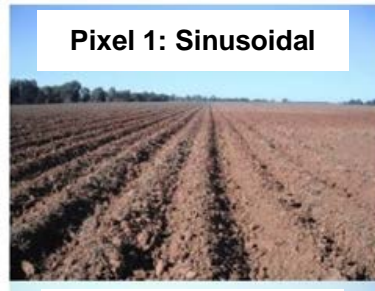

Pixel 2: Flat bench

(b)

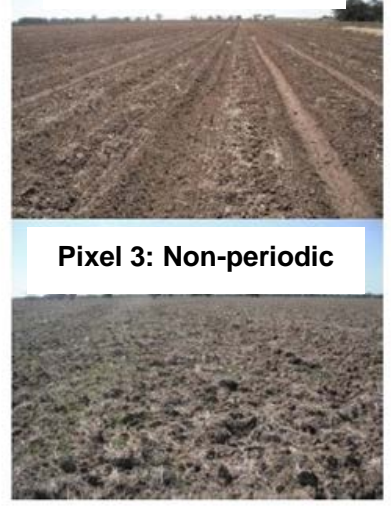

Figure 1. (a) Map of the Yanco Region showing the 3 selected bare pixels (zoomed in). Red boxes indicate the 6 focus areas; red triangles indicate the roughness sampling locations, and arrows show the look direction of PLIS. (b) Picture showing the dominant surface roughness pattern of the three bare pixels.

\subsection{Airborne data}

The Polarimetric L-band Multibeam Radiometer (PLMR) and Polarimetric L-band Imaging Synthetic aperture radar (PLIS) were simultaneously flown on 9 different days during the 3-week campaign. At a flight altitude of 3,000m above the ground, radiometer brightness temperature $\left(T_{B}\right)$ and radar backscatter coefficient $\left(\sigma^{0}\right)$ were acquired at $1 \mathrm{~km}$ and $10 \mathrm{~m}$ resolution respectively, over the $36 \mathrm{~km} \times 38 \mathrm{~km}$ regional area in Fig. 1 , with incidence angle ranging from $7^{\circ}-38.5^{\circ}$ for PLMR and $15^{\circ}-45^{\circ}$ for PLIS. For the data used in this study, $T_{B}$ has been normalized to the soil profile temperature in the middle of the flight period and to an incidence angle of $38.5^{\circ}$. Likewise, $\sigma^{\circ}$ has also been normalized to an angle of $40^{\circ}$. Incidence angle normalization of both data sets has followed the technique in (Ye et al., 2013). Since $\sigma^{0}$ and $T_{B}$ do not have the same spatial resolution, $\sigma^{0}$ data have been aggregated from $10 \mathrm{~m}$ to $1 \mathrm{~km}$ by averaging the values within that $1 \mathrm{~km}$ pixel in linear units, for ease of comparison with passive microwave data.

\subsection{Ground sampling data}

Soil moisture sampling was undertaken concurrently with each regional flight during the experiment. The sampling was undertaken with a Steven's Water Hydraprobe following a regular grid of 250m-spaced locations, with two of the focus areas measured during each of the flights. Three soil moisture measurements were made per location to minimise the effect of sampling errors.

Surface roughness was also measured within each major land cover type at the six focus areas. At each measurement location, two 3m-long surface profiles were recorded; one oriented parallel to the look direction of PLIS (East-West) and one perpendicular (North-South). When periodic row structures were observed, profiles were orientated in the across and along direction with respect to the tillage instead, and the row orientation recorded. In these cases, a Fourier transform analysis of the roughness data was performed to separate the small-scale from the large-scale roughness. A Fourier separation factor $K(\mathrm{rad} / \mathrm{m})$ was adjusted to capture the periodic structure and to filter the large-scale roughness spectrum out from the profile. In this study, only the small-scale roughness in the East-West direction ( $K$ ranges from 7 to $20 \mathrm{rad} / \mathrm{m}$ ) was used, because $\sigma^{0}$ has been found to be highly correlated with high-frequency roughness (Fung, 1994) and EastWest is the look direction of the PLIS sensor. 


\section{MODEL DESCRIPTION}

\subsection{Passive microwave model}

The bare soil microwave emission is a function of the ground emissivity (or reflectivity) and the soil effective temperature as:

$$
T_{B P}=e_{P} \cdot T_{E F F}=\left(1-r_{P}\right) T_{E F F},
$$

where $T_{E F F}(\mathrm{~K})$ is the effective soil temperature; $e(-)$ is the soil emissivity; $r(-)$ is the soil reflectivity and subscript $P$ indicates polarization (horizontal or vertical). The soil reflectivity is computed from the soil roughness parameters $H_{R}(-)$ and $N_{R P}(-)$ as:

$$
r=r^{*} \cdot \exp \left[-H_{R} \cos (\vartheta)^{N_{R P}}\right],
$$

where the smooth soil reflectivity $r^{*}$ is related to the surface soil moisture content through the Fresnel equations and a dielectric constant model; $\vartheta$ is the incidence angel (degree). In this paper, $N_{R}$ equals to 1 and 1 for h- and v-polarization respectively according to (Wigneron et al., 2007). The models proposed in Dobson et al. (1985) and (Mironov et al., 2004) have been tested in this study, and Dobson's model was finally selected since it provided slightly better retrieval accuracy.

The soil effective temperature is determined as a function of two temperature measurements: one at the soil surface $(2-5 \mathrm{~cm}) T_{\text {SURF }}\left({ }^{\circ} \mathrm{C}\right)$, the other at a greater depth $(\sim 50 \mathrm{~cm}) T_{D E P T H}\left({ }^{\circ} \mathrm{C}\right)$ as:

$$
T_{E F F}=T_{D E P T H}+\left(T_{S U R F}-T_{D E P T H}\right) \cdot\left(M_{v} / w_{0}\right)^{b_{0}},
$$

where $M_{v}$ is the surface soil moisture $\left(\mathrm{m}^{3} / \mathrm{m}^{3}\right)$ at about $0-2 \mathrm{~cm}$, and $w_{0}$ and $b_{0}$ are semi-empirical parameters depending on specific soil characteristics. Parameters $w_{0}=0.398$ and $b_{0}=0.181$ were calibrated from a study with similar soil properties (Wigneron et al., 2008) and applied in this study. $T_{\text {SURF }}$ and $T_{D E P T H}$ were obtained from in-situ monitoring stations.

\subsection{Active microwave model}

The semi-empirical model by (Oh, 2004) was chosen as the active model for a bare surface. Semi-empirical models combine the theoretical background with simulated or experimental data sets, and thus are not expected to have the same site-specific restrictions as the empirical models, while they simplify the complexity of the theoretical models. The Oh (2004) model is written as:

$$
\begin{aligned}
& \sigma^{0}{ }_{v h}=0.11 M_{v}^{0.7}(\cos \vartheta)^{2.2}\left\{\left[1-\exp \left[-0.32(k s)^{1.8}\right]\right\},\right. \\
& p=\sigma_{h h}^{0} / \sigma_{v v}^{0}=1-\left(\frac{\vartheta}{90^{\circ}}\right)^{0.35 M v^{-0.65}} \cdot e^{-0.4(k s)^{1.4},} \\
& q=\sigma_{v h}^{0} / \sigma^{0}{ }_{v v}=0.095(0.13+\sin 1.5 \vartheta)^{1.4}\left\{1-\exp \left[-1.3(k s)^{1.8}\right]\right\},
\end{aligned}
$$

where $\sigma_{v h}^{0}, \sigma_{h h}^{0}$ and $\sigma_{v v}^{0}$ are the vh-, hh- and vv-polarized backscattering coefficients (in power units) respectively; $p$ is the co-polarized ratio and $\vartheta$ is the incidence angle (degree); $M_{v}$ is the volumetric soil moisture content; $s$ is the Root-Mean-Square (RMS) soil surface roughness height and $k$ is the wavenumber $(k=2 \pi f / c)$. It should be noted that the model has three equations in total, and thus has redundant solutions. However Equation 6 was not able to obtain a real number solution for most of the PLIS data within the selected pixels. Thus only the first two equations were applied.

\subsection{Retrieval and method}

In the passive microwave model, the soil moisture and the roughness parameter $H_{R}$ were considered to be unknown variables and retrieved from PLMR observations of $T_{B V}$ and $T_{B H}$ by directly inverting the model in Equation 1. Similarly, using Equations 4 and 5 the soil moisture $M_{v}$ and the RMS height $s$ were considered unknown and estimated from radar observations of $\sigma_{v h}^{0}, \sigma_{h h}^{0}$ and $\sigma_{v v}^{0}$.

To simplify the comparison between active-retrieved and passive-retrieved roughness, the active-retrieved $s$ was converted into "effective roughness" $H_{R}$, based on Equation 7 (Choudhury et al., 1979) and Equation 8 (Wigneron et al., 2011), respectively:

$$
\begin{aligned}
& H_{R}=(2 k s)^{2}, \\
& H_{R}=(0.9437 s /(0.8865 s+2.2913))^{6},
\end{aligned}
$$

where $k$ is the wave number and $s$ is the surface RMS height. 


\section{RESULTS AND DISCUSSION}

The retrieved soil moisture from active and passive microwave observations was compared with the ground sampling, as shown in Figure 2. It can be seen that soil moisture estimates from passive measurements have a better accuracy $\left(\mathrm{RMSE}=0.05 \mathrm{~m}^{3} / \mathrm{m}^{3}\right)$ than those from active measurements $\left(\mathrm{RMSE}=0.11 \mathrm{~m}^{3} / \mathrm{m}^{3}\right)$. It was noted that estimates from PLIS observations significantly underestimated the soil moisture $\left(\right.$ Bias $=-0.08 \mathrm{~m}^{3} / \mathrm{m}^{3}$ ), especially in the higher $\left(0.15-0.35 \mathrm{~m}^{3} / \mathrm{m}^{3}\right)$ soil moisture range. This result reconfirms that passive microwave sensors are more suitable in estimating soil moisture than active sensors and perform better in retrieving accurate soil moisture values, regardless of its low resolution.

Figure 3 shows the evaluation of retrieved surface RMS height from active measurements. The horizontal scatter associated with points having the same color represents RMS retreived within the same pixel but from different flights. This shows that even within the same pixel, the retreived roughness on different days can range from around $1 \mathrm{~cm}$ to $2 \mathrm{~cm}$. This is attributed to possible retreival errors from 1) $\sigma^{0}$ aggregation, 2) incidence angle normalisation and 3) instrument error. Apart from these, the observed RMS heights were assumed to be consistent throughout the experiment period. However there might be small changes in reality. Nevertheless, when compared with the in-situ RMS height, it is clear that the non-periodic pixel has the best retreival accuracy $\left(0.35 \mathrm{~m}^{3} / \mathrm{m}^{3}\right)$, although it also has the largest sampling standard deviation $(1.07 \mathrm{~cm})$. However, for the two pixels with periodic structure, the retreived values tend to overestimate the surface roughness (Bias $=0.45 \mathrm{~cm}$ and $0.49 \mathrm{~cm}$ respectively). This could result from the processing of in-situ roughness sampling. As mentioned, the large-scale roughness of these periodic structures has been removed by applying a separation factor $K$, since radar is more sensitive to the small-scale roughness. However, it is hard to differentiate between the 'small-scale' and 'large scale', and thus to determine the factor $K$. Currently no researches have addressed this scale problem for radar measurements. This means that the correlation between radar observation and the level of roughness scale (especially for periodic structures) requires further study.

In order to assess the feasibility of using the active-retrieved roughness parameter in passive soil moisture retrieval, the active-retrieved surface RMS height was converted into the passive roughness parameter $H_{R}$, and then compared with the $H_{R}$ retrieved from passive measurements. As described, the empirical equations given by Equations 7 and 8 were applied for the conversion. Results for all three types of roughness profiles are shown in Figure 4. There is no clear evidence of the impact of structure patterns on the results. Although the similarity between the two types of $H_{R}$ is slightly higher when using Choudhury's equation, the active- $H_{R}$ estimated from both equations still tend to underestimate the passive- $H_{R}$. This result suggests that 'roughness' has a different physical meaning in active microwave and passive microwave remote sensing. Unlike the

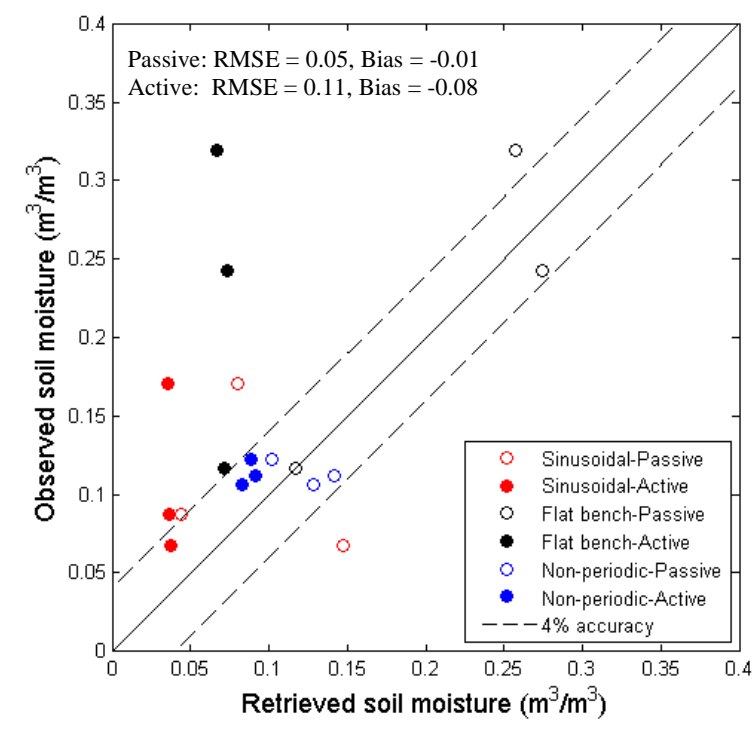

Figure 2. Comparison between retrieved soil moisture from active and passive observations and in-situ soil moisture. The RMSE for active and passive retrieval is $0.11 \mathrm{~m}^{3} / \mathrm{m}^{3}$ and $0.05 \mathrm{~m}^{3} / \mathrm{m}^{3}$ respectively. Black dashed lines indicated the SMAP soil moisture target accuracy $\left(0.04 \mathrm{~m}^{3} / \mathrm{m}^{3}\right)$.

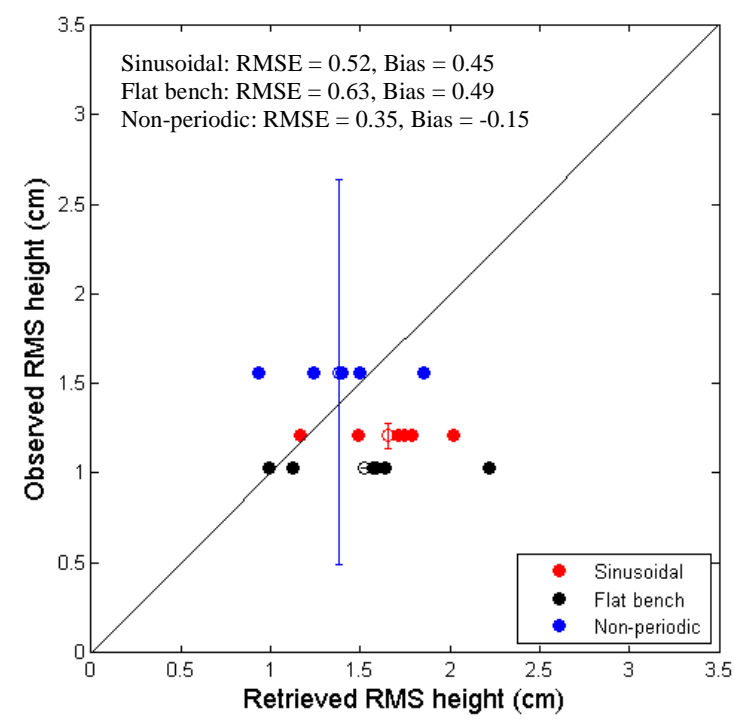

Figure 3. Evaluation of the retrieved RMS height from active observations only. Observed RMS was averaged from samples within each $1 \mathrm{~km}$ pixel. Whiskers indicate sampling standard deviation. Note that there is no whisker for the flat bench since only 1 sample is available. 

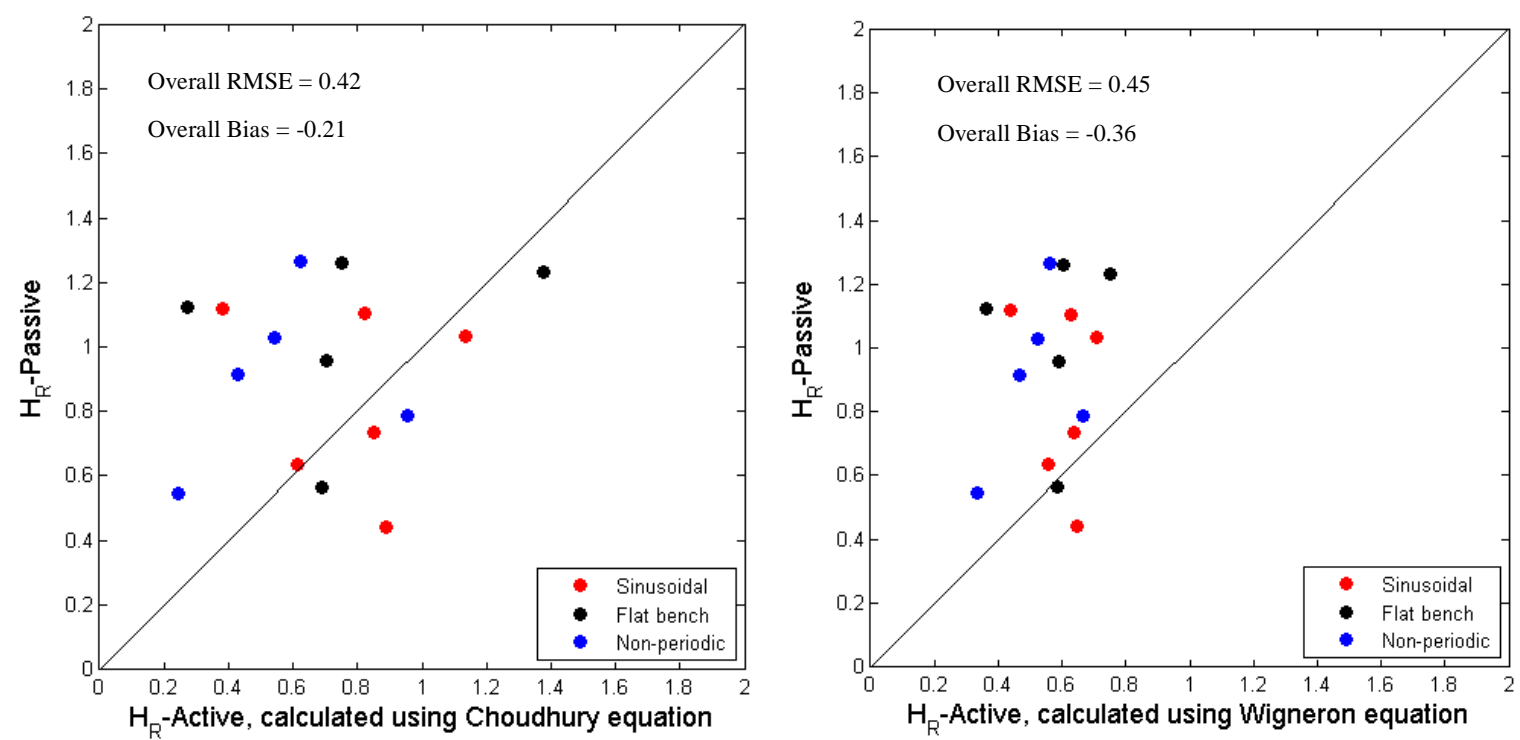

Figure 4. Comparison between $H_{R}$ parameter retrieved from passive microwave and $H_{R}$ calculated from active-retrieved surface RMS height, using Equation 7 (left) and Equation 8 (right).

surface RMS height which is used in active model, $H_{R}$ in the passive model is more of an effective parameter which is not physically measurable. As also discussed in (Choudhury et al., 1979) it has been a difficult job to find a not only general but also numerically accurate quantitative description for $H_{R}$. Moreover, according to the results in Figure 4 that the active- $H_{R}$ tends to underestimate the passive- $H_{R}$, it can be inferred that passive microwave should be more sensitive to roughness in larger-scale (i.e. greater surface RMS height), compared to active microwave. However, future studies involving more bare pixels and ground sampling data are needed to confirm this statement.

\section{CONCLUSION}

This study performed a comparison between surface roughness parameters retrieved from active and passive microwave measurements over a bare soil surface, using data from the third Soil Moisture Active Passive Experiment (SMAPEx-3) conducted in Australia in 2011. The main purpose of this study was to assess whether the roughness parameters derived from active microwave data can be used directly in passive microwave retrievals. A number of conclusions can be drawn from this study: 1) Compared with active microwave, passive microwave observations provided a more accurate soil moisture estimation; 2) the correlation between radar observation and the level of roughness scale (especially for periodic structures) requires further study; 3) 'Roughness' seems to have a different physical meaning in active microwave and passive microwave remote sensing; and 4) Passive microwave may be more sensitive to large-scale roughness compared with active microwave. However, studies involving more data are required to confirm these points and will be conducted in the future.

\section{ACKNOWLEDGMENTS}

The authors would like to thank all the SMAPEx-3 participants, the research funding from the Australian Research Council (DP0984586, LE0453434 and LE0882509) and the postgraduate scholarship for Ying Gao from Faculty of Engineering, Monash University.

\section{REFERENCES}

Choudhury, B. J., T. J. Schmugge, A. Chang and R. W. Newton (1979). Effect of Surface-Roughness on the Microwave Emission from Soils. Journal of Geophysical Research-Oceans and Atmospheres 84(NC9): 5699-5706. 
Entekhabi, D., E. G. Njoku, P. E. O'Neill, K. H. Kellogg, W. T. Crow, W. N. Edelstein, J. K. Entin, S. D. Goodman, T. J. Jackson, J. Johnson, J. Kimball, J. R. Piepmeier, R. D. Koster, N. Martin, K. C. McDonald, M. Moghaddam, S. Moran, R. Reichle, J. C. Shi, M. W. Spencer, S. W. Thurman, L. Tsang and J. Van Zyl (2010). The Soil Moisture Active Passive (SMAP) Mission. Proceedings of the IEEE 98(5): 704-716.

Fung, A. K. (1994). Microwave scattering and emission models and their applications. Boston, Artech House.

Kerr, Y. H., P. Waldteufel, J. P. Wigneron, S. Delwart, F. Cabot, J. Boutin, M. J. Escorihuela, J. Font, N. Reul, C. Gruhier, S. E. Juglea, M. R. Drinkwater, A. Hahne, M. Martin-Neira and S. Mecklenburg (2010). The SMOS Mission: New Tool for Monitoring Key Elements ofthe Global Water Cycle. Proceedings of the IEEE 98(5): 666-687.

Mironov, V. L., M. C. Dobson, V. H. Kaupp, S. A. Komarov and V. N. Kleshchenko (2004). Generalized refractive mixing dielectric model for moist soils. IEEE Transactions on Geoscience and Remote Sensing 42(4): 773-785.

Njoku, E. G. and D. Entekhabi (1996). Passive microwave remote sensing of soil moisture. Journal of Hydrology 184(1-2): 101-129.

Oh, Y. (2004). Quantitative retrieval of soil moisture content and surface roughness from multipolarized radar observations of bare soil surfaces. IEEE Transactions on Geoscience and Remote Sensing 42(3): 596-601.

Panciera, R., J. P. Walker, T. J. Jackson, D. Gray, M. A. Tanase, D. Ryu, A. Monerris, H. Yardley, C. Rüdiger, X. Wu, Y. Gao and J. Hacker (2013). The Soil Moisture Active Passive Experiments (SMAPEx): Towards Soil Moisture Retrieval from the SMAP Mission. IEEE Transaction on Geoscience and Remote Sensing (In Press).

Panciera, R., J. P. Walker and O. Merlin (2009). Improved Understanding of Soil Surface Roughness Parameterization for L-Band Passive Microwave Soil Moisture Retrieval. IEEE Geoscience and Remote Sensing Letters 6(4): 625-629.

Saleh, K., Y. H. Kerr, P. Richaume, M. J. Escorihuela, R. Panciera, S. Delwart, G. Boulet, P. Maisongrande, J. P. Walker, P. Wursteisen and J. P. Wigneron (2009). Soil moisture retrievals at L-band using a two-step inversion approach (COSMOS/NAFE'05 Experiment). Remote Sensing of Environment 113(6): 1304-1312.

Schmugge, T. J. (1985). Chapter 5: Remote Sensing of Soil Moisture, In: Anderson, M. G., and Burt, T. P. (Eds.). New York, John Wiley and Sons.

Wigneron, J. P., A. Chanzy, P. de Rosnay, C. Rüdiger and J. C. Calvet (2008). Estimating the effective soil temperature at L-band as a function of soil properties. IEEE Transactions on Geoscience and Remote Sensing 46(3): 797-807.

Wigneron, J. P., A. Chanzy, Y. H. Kerr, H. Lawrence, J. Shi, M. J. Escorihuela, V. Mironov, A. Mialon, F. Demontoux, P. de Rosnay and K. Saleh-Contell (2011). Evaluating an Improved Parameterization of the Soil Emission in L-MEB. IEEE Transactions on Geoscience and Remote Sensing 49(4): 11771189.

Wigneron, J. P., Y. Kerr, P. Waldteufel, K. Saleh, M. J. Escorihuela, P. Richaume, P. Ferrazzoli, P. de Rosnay, R. Gurney, J. C. Calvet, J. P. Grant, M. Guglielmetti, B. Hornbuckle, C. Matzler, T. Pellarin and M. Schwank (2007). L-band Microwave Emission of the Biosphere (L-MEB) Model: Description and calibration against experimental data sets over crop fields. Remote Sensing of Environment 107(4): 639-655.

Ye, N., J. P. Walker and C. Rüdiger (2013). A Cumulative Distribution Function Method for Normalising Multi-angle Microwave Observations. IEEE Transaction on Geoscience and Remote Sensing: In Review. 